\title{
Effectiveness and tolerability of intravenous pentamidine for Pneumocystis carinii pneumonia prophylaxis in adult hematopoietic stem cell transplant patients: a retrospective study
}

\author{
Wedad B. Awad*i(1), Alaa Asaad, Nardin Al-Yasein and Rula Najjar
}

\begin{abstract}
Background: Pneumocystis carinii pneumonia (PCP) prophylaxis is recommended after hematopoietic stem cell transplantation (HSCT). In patients who are unable to take first-line prophylaxis, trimethoprim/sulfamethoxazole, aerosolized pentamidine is recommended. This drug may not, however, be available at all institutions, and its administration requires special techniques. Therefore, intravenous pentamidine (IVP) has been used in adult patients as an alternative, despite limited data. We evaluated the effectiveness and tolerability of IVP for PCP prophylaxis in adult patients who had undergone HSCT.

Methods: A single-center retrospective study was conducted of adult patients who had undergone allogenic or autologous HSCT between January 2014 and September 2018 and had received at least three doses of IVP for PCP prophylaxis. The IVP dose was $4 \mathrm{mg} / \mathrm{kg}$ administered monthly. Data on PCP infection and adverse reactions were collected from both patients' electronic medical records and the pharmacy adverse drug reactions documentation system. Patients were followed from the start of IVP up to 6 months after discontinuation of therapy. A confirmed PCP infection was defined as radiographic evidence of PCP and positive staining of a respiratory specimen. Descriptive statistics were used to analyze the study outcomes.

Results: During the study period, 187 patients were included. The median age was 36.4 years (range, 18-64), 58\% were male, and 122 (65\%) had received allogeneic HSCT while the remainder autologous HSCT. The median number of IVP doses administered per patient was 5 (range, 3-29). During the study period, none of the patients had evidence of confirmed PCP infection. However; there were two cases with high clinical suspicion of PCP infection (i.e. required anti-pneumocystis therapy) and one reported case of central nervous system toxoplasmosis while receiving IVP for PCP prophylaxis. Only one case of nausea associated with IVP administration was reported.

* Correspondence: WA.12503@khcc.jo

Department of Pharmacy, King Hussein Cancer Center, P.O. Box 1269,

Al-Jubeiha, Amman 11941, Jordan

(c) The Author(s). 2020 Open Access This article is licensed under a Creative Commons Attribution 4.0 International License, which permits use, sharing, adaptation, distribution and reproduction in any medium or format, as long as you give appropriate credit to the original author(s) and the source, provide a link to the Creative Commons licence, and indicate if changes were made. The images or other third party material in this article are included in the article's Creative Commons licence, unless indicated otherwise in a credit line to the material. If material is not included in the article's Creative Commons licence and your intended use is not permitted by statutory regulation or exceeds the permitted use, you will need to obtain permission directly from the copyright holder. To view a copy of this licence, visit http://creativecommons.org/licenses/by/4.0/ The Creative Commons Public Domain Dedication waiver (http://creativecommons.org/publicdomain/zero/1.0/) applies to the data made available in this article, unless otherwise stated in a credit line to the data. 
(Continued from previous page)

Conclusions: In a cohort of adult patients with HSCT who received IVP for PCP prophylaxis, there was no evidence of confirmed PCP infection, and the treatment appeared to be well tolerated. Prospective studies should be conducted to confirm the efficacy and tolerability of IVP.

Keywords: Pentamidine, Prophylaxis, PCP, Pneumocystis pneumonia, Pneumocystis jirovecii, Hematopoietic stem cell transplantation

\section{Background}

Pneumocystis jirovecii (formerly carinii) pneumonia (PCP), is a serious fungal opportunistic infection that affects immunocompromised patients. The mortality rate associated with PCP infections in non-HIV highrisk patients is $30-60 \%[1,2]$. The expected risk of PCP after allogeneic hematopoietic stem cell transplantation (HSCT) is $5-15 \%$ in the absence of prophylaxis [3, 4], with a mortality rate of up to $89 \%$ in patients who develop PCP within 6 months of allogeneic HSCT and reaches up to $40 \%$ after 6 months from allogenic HSCT $[3,5]$.

Advances in prophylactic and treatment modalities have improved the outcome of PCP infection over the years. The guidelines of the American Society for Blood and Marrow Transplant, the Centers for Disease Control and Prevention and the European Conference on Infections in Leukemia recommend routine PCP prophylaxis in immunocompromised patients, including patients after HSCT [6-8].

The risk of infection in patients who undergo HSCT is determined primarily by the type of transplant (autologous, allogeneic), the time since transplantation, the presence or absence of graft-versus-host disease (GVHD), donor-host histocompatibility, disease status, graft type, graft content, conditioning intensity and neutrophil engraftment [6]. PCP prophylaxis is recommended for at least the initial 6 months after allogeneic HSCT [9], in patients with active GVHD who require treatment and in patients with hematological relapse or on corticosteroid use [6]. In autologous HSCT recipients, PCP prophylaxis is typically given for 3-6 months after transplantation and longer for those receiving immunosuppressive drugs [6].

Trimethoprim/sulfamethoxazole (TMP/SMX) is firstline prophylaxis against PCP after HSCT $[10,11]$; however, intolerance to TMP/SMX in HSCT recipients has been reported to be as high as 55\%, requiring discontinuation of the drug due to myelosuppression, rash or allergy [12]. Dapsone and aerosolized pentamidine are considered second-line therapy; they do not cause myelosuppression and are considered effective alternatives as prophylaxis against PCP infections in HSCT recipients. Dapsone may, however, cause hemolytic anemia, and aerosolized pentamidine may not be available at all institutions and requires special administration techniques and staff training that is not available at many centers, such as our institution. In addition, the cost of administration of aerosolized pentamidine is higher than that of oral therapies, and it may cause bronchospasm or dyspnea and has been associated with atypical manifestations of infection, such as atypical pneumonia and extrapulmonary disease $[10,11]$.

Intravenous pentamidine (IVP) is approved by the United States Food and Drug Administration in pediatrics for both treatment and prophylaxis of PCP, while the European Medicines Agency approves it only for treatment of PCP. In adults, IVP is approved by both agencies only for the treatment of PCP, and limited available data are available to support its use in prophylaxis. It has no myelosuppressive effect and few of the adverse effects reported with inhaled pentamidine [13-15]. Given these data, we evaluated the effectiveness and tolerability of monthly IVP for prophylaxis in adult HSCT recipients.

\section{Methods}

A retrospective study was conducted at King Hussein Cancer Center (KHCC), an internationally accredited comprehensive cancer teaching hospital in Amman, Jordan, that provides care for both adult and pediatric patients. The center accommodates a specialized adult bone marrow transplant unit, where an average of 100 transplants are performed per year.

The study included adult patients ( $\geq 18$ years) who underwent allogeneic or autologous HSCT between January 2014 and September 2018 and received at least three consecutive doses of IVP for prophylaxis between the day of transplantation and 1 year post transplantation. Patients were excluded if they were pregnant.

According to our hospital's protocol, all HSCT patients receive PCP prophylaxis post engraftment. TMPSMX is standardly used as first line drug of choice. In case of poor hematopoietic engraftment or inability to tolerate oral medications, patients are started then on IVP or dapsone. In addition, patients who develop intolerance or myelosuppression with TMP-SMX use, they are switched to IVP. Duration of prophylaxis is 6 months for autologous recipients and at least 1 year for allogenic recipients. IVP for PCP prophylaxis is given as a monthly dose of $4 \mathrm{mg} / \mathrm{kg}$ of body weight infused over a minimum of $1 \mathrm{~h}$, with ranitidine, hyoscine and/or metoclopramide given intravenously as pre-medication. The 
specific regimen for pre-medication is based on clinical judgement.

Patient data was obtained from the bone marrow transplant unit database and electronic patient profiles. The list of HSCT patients who received IVP was extracted from the pharmacy billing system for the study period, filtered according to the number of IVP doses received and then matched with the adult bone marrow transplant unit database. The data were then entered into a de-identified, secured central database.

PCP infection work-up is performed for any patient with respiratory symptoms after consultation with the adult infectious disease specialist. The workup consisted of obtaining respiratory specimen through bronchoalveolar lavage for those with suspected PCP infection based on the chest computed tomography scan. PCP infection was identified by reviews of patients' electronic medical records for any clinical, radiographic or microbiological evidence, and positive (dye-based) or fluorescent antibody staining of respiratory specimens. Any PCP infection identified after the start of IVP prophylaxis was considered as failure of the regimen. Patients were followed-up for at least 6 months after discontinuation of IVP. Furthermore, Patients' electronic medical profiles were evaluated for any evidence of toxoplasmosis infection while on IVP for PCP prophylaxis.

The incidence of adverse drug reactions after administration of IVP was obtained from both patients' electronic profile reviewed for 1 week after each administered dose of IVP and the pharmacy online documentation system for adverse drug reactions.

Descriptive statistics were used to analyze all background and patient demographic data as well as the effectiveness and tolerability outcomes. Categorical data were reported as absolute and relative frequencies, while continuous data were reported as means and standard deviations or medians and ranges.

\section{Results}

A total of 454 patient who received pentamidine during the study period were identified. Among those, 183 patients received less than 3 doses of pentamidine, and therefore were excluded from the cohort. For those patients, IVP was switched to oral TMP-SMX due to improvement in their hematological counts and ability to continue on first line therapy as per our institution's standard protocol. None of them were stopped due to development of PCP or intolerance to pentamidine. Of the remaining 271 transplant recipients, 187 met the inclusion criteria. The remaining 84 patients were excluded as they either did not undergo HSCT and/or they are less than 18 years of age (Fig. 1). All 187 patients received a dose of $4 \mathrm{mg} / \mathrm{kg}$, and 182 received one or more of the pre-medications. Patient baseline characteristics included demographics, primary diagnosis, conditioning regimen intensity, transplant stem cell source, HLA matching for patients undergoing allogeneic transplant, incidence of acute and chronic GVHD, and relevant comorbidity (Table 1).

Of the 187 patients, 122 (65.2\%) had undergone allogeneic bone marrow transplantation and 65 patients (34.8\%) autologous bone marrow transplantation. The two primary indications for HSCT were leukemia and lymphoma, representing 41.2 and $35.3 \%$ of the patients; respectively. HSCT was performed in $14.9 \%$ of the patients for a non-malignant condition.

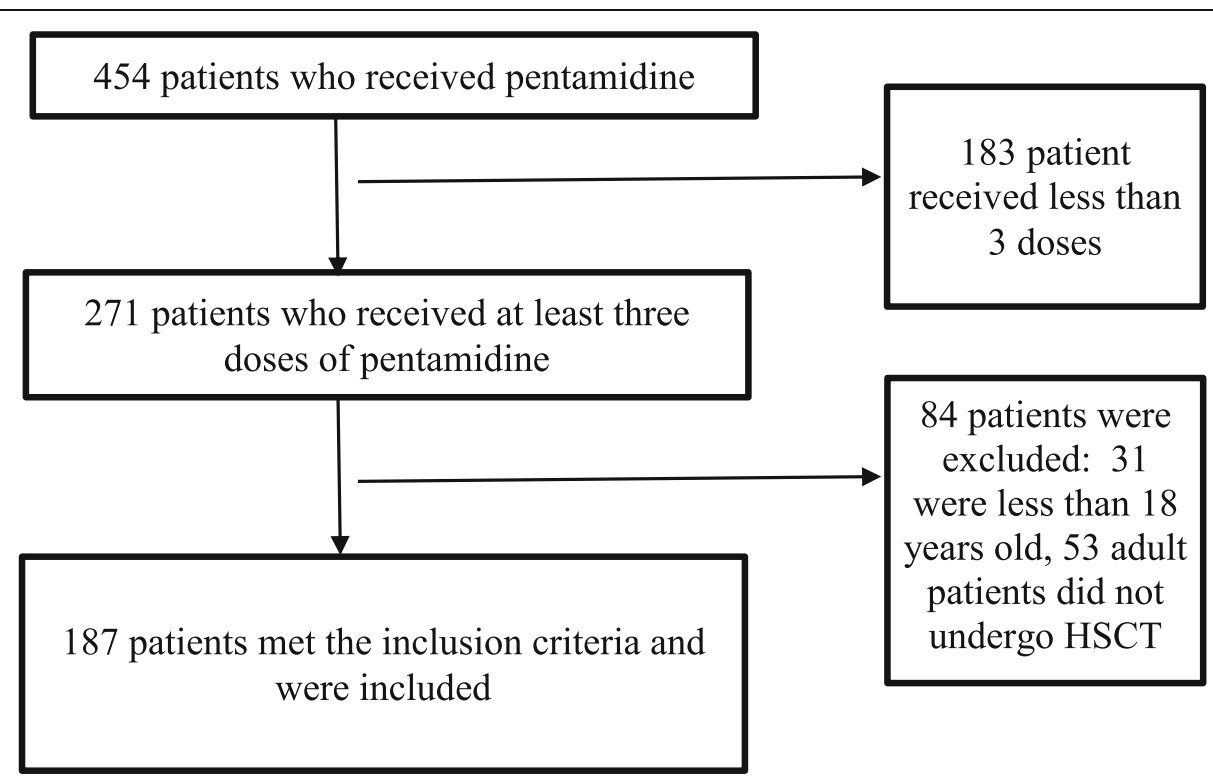

Fig. 1 Flow diagram of included patients 
Table 1 Baseline characteristics of patients

\begin{tabular}{|c|c|}
\hline Baseline characteristic & Value $(N=187$ \\
\hline Age (years), median (range) & $36.4(18-64)$ \\
\hline \multicolumn{2}{|l|}{ Gender, N (\%) } \\
\hline Male & $108(57.7 \%)$ \\
\hline \multicolumn{2}{|l|}{ Primary diagnosis, N (\%) } \\
\hline Leukaemia & $77(41.2 \%)$ \\
\hline Acute lymphoblastic leukaemia & 19 \\
\hline Acute myeloid leukaemia & 39 \\
\hline Chronic myeloid leukaemia & 7 \\
\hline Myelodysplastic syndrome & 12 \\
\hline Lymphoma & $66(35.3 \%)$ \\
\hline Non-Hodgkin lymphoma & 24 \\
\hline Hodgkin lymphoma & 42 \\
\hline Other malignancies & $16(8.5 \%)$ \\
\hline Multiple myeloma & 15 \\
\hline Germ-cell tumour & 1 \\
\hline Non-malignancies & $28(14.9 \%)$ \\
\hline Aplastic anaemia & 14 \\
\hline Fanconi anaemia & 1 \\
\hline Myelofibrosis & 2 \\
\hline Red-cell aplasia & 1 \\
\hline Sickle-cell anaemia & 1 \\
\hline Thalassaemia & 9 \\
\hline \multicolumn{2}{|l|}{ Type of transplant } \\
\hline Autologous & $65(34.8 \%)$ \\
\hline Allogeneic & $122(65.2 \%)$ \\
\hline Related donor & 15 \\
\hline Unrelated donor & 105 \\
\hline Haplo-identical transplant & 2 \\
\hline \multicolumn{2}{|l|}{ Stem cell source, N (\%) } \\
\hline Peripheral stem cells & $166(88.8 \%)$ \\
\hline Bone marrow cells & $19(10.1 \%)$ \\
\hline Bone marrow and peripheral stem cells & $1(0.5 \%)$ \\
\hline Umbilical cord bank & $1(0.5 \%)$ \\
\hline \multicolumn{2}{|l|}{ Conditioning regimen intensity, N (\%) } \\
\hline Myeloablative & $130(69.5 \%)$ \\
\hline Reduced intensity & $55(29.4 \%)$ \\
\hline Reduced toxicity & $1(0.5 \%)$ \\
\hline None & $1(0.5 \%)$ \\
\hline \multicolumn{2}{|l|}{ Baseline comorbidity, N (\%) } \\
\hline Cardiac disease & $40(21.4 \%)$ \\
\hline Endocrine disease & $25(13.3 \%)$ \\
\hline Kidney disease & $10(5.3 \%)$ \\
\hline Liver disease & $6(3.2 \%)$ \\
\hline Other & $43(22.9 \%)$ \\
\hline
\end{tabular}

Table 1 Baseline characteristics of patients (Continued)

\begin{tabular}{ll}
\hline Baseline characteristic & Value $(N=187)$ \\
\hline GVHD, N (\%) & $16(8.6 \%)$ \\
Acute & $31(16.6 \%)$ \\
Chronic & $38(20.3 \%)$ \\
Both acute and chronic & $102(54.5 \%)$ \\
None & \\
\hline
\end{tabular}

The majority of patients (82.4\%) received up to eight monthly doses of IVP prophylaxis; $11.2 \%$ continued the prophylaxis for $9-12$ doses and $6.4 \%$ for $>1$ year. The median number of doses was 5 (range, 3-29) (Table 2). Patients were started on IVP at a mean of day 31 posttransplant, with a median of 25 days (range, 1-127). During the study period, none of the patients had evidence of confirmed PCP infection. Although 32 patients were evaluated for suspected PCP; none had confirmed diagnosis and only two patients continued to receive treatment despite negative respiratory specimens due to a high clinical suspicion of PCP. Only one patient was reported to have central nervous system toxoplasmosis while receiving IVP for PCP prophylaxis.

IVP was used as the first choice for PCP prophylaxis for 136 patients (72.7\%); the others were started initially on TMP/SMX or dapsone per standard care and then switched to IVP due to intolerability.

Five patients received no pre-medication before IVP administration; none had a documented adverse drug reaction. Among the study population, only one patient reported nausea as an adverse event after administration of IVP despite receiving proper pre-medications.

Table 2 Pentamidine-related data

\begin{tabular}{ll}
\hline Variable & Value \\
\hline Number of pentamidine doses, median (range) & $5(3-29)$ \\
$3-5$ & 106 \\
$6-8$ & 48 \\
$9-12$ & 21 \\
$>12$ & 12 \\
Complete blood count at first pentamidine dose, N (\%) & \\
Normal ANC ${ }^{\text {a }}$ and platelet count & $80(42.6 \%)$ \\
Low ANC ${ }^{a}$ and platelet count & $96(51.6 \%)$ \\
Could not be obtained & $11(5.9 \%)$ \\
Day of IVP initiation after transplantation, median (range) & $25(1-127)$ \\
Choice of IVP for prophylaxis, N (\%) & $136(72.7 \%)$ \\
First choice & $51(27.3 \%)$ \\
Second choice &
\end{tabular}

${ }^{\mathrm{a}}$ ANC Absolute neutrophil count 


\section{Discussion}

This retrospective study supports the use of IVP in adult HSCT patients with no documented confirmed PCP infections. Although TMP-SMX remains the first line drug choice for prophylaxis against PCP, given its effectiveness against Pneumocystis jirovecii and other opportunistic infections, as well as its relatively low cost, second line agents such as IVP may be necessary in cases of intolerance, sulfa allergy and myelosuppression.

Regarding dosing of IVP, the administered IVP dose in our study was $4 \mathrm{mg} / \mathrm{kg}$ per month infused over a minimum of $1 \mathrm{~h}$ after pre-medication with IV ranitidine, hyoscine and/or metoclopramide. Sweiss et al. [16] reported an IVP dose of $4 \mathrm{mg} / \mathrm{kg}$ (with a maximum of $300 \mathrm{mg}$ per dose) infused over a standard infusion time of $2 \mathrm{~h}$ and the pre-medication was ondansetron in their study. Another study, Diri $\mathrm{R}$ et al. [3], reported use of a standard IVP dose of $300 \mathrm{mg}$ and pre-medication with diphenhydramine and ondansetron before infusion. Furthermore, our patients were started on IVP at a mean of 31 days after transplant, while in comparison with other studies [3, 16] IVP was started at any time after the end of conditioning chemotherapy or within 6 days of the scheduled allogeneic transplantation.

The incidence of PCP infection in adult HSCT patients who received IVP for prophylaxis was reported in two studies $[3,16]$, one prospective and the other retrospective. Both studies reported no PCP infection, as in our study. The study population in the prospective study consisted of adults who had undergone HSCT or had received only intensive chemotherapy, and the retrospective study included only patients who had undergone allogeneic HSCT patients. These findings are consistent with those reported in the literature in pediatric HSCT population [11, 13, 17, 18]. However, a concern toward an increased risk of breakthrough PCP infection in younger patients receiving IVP as PCP prophylaxis was reported in the pediatric population [11].

In term of IVP tolerability, injection site reaction, renal insufficiency, hypotension, gastrointestinal discomfort, leukopenia, azotemia, increased liver enzymes, skin rash and flushing were possible adverse events reported in the literature following IVP administration $[19,20]$. We had only one documented adverse event among our patients that was nausea. Sweiss et al. [16] reported in their prospective study that nausea (8\%) and hypotension (12\%) as common adverse events in their patients, and less commonly nasal congestion (4\%), oral numbness (4\%), infusion related reaction (4\%), acute kidney injury (4\%) and rash (2\%). Nevertheless, all reported adverse events were of grade I/II and none had reported grade III/IV adverse events. In pediatrics, DeMasi J et al. [18] reported nausea/vomiting $(7.3 \%)$, grade IV reaction with anaphylaxis (rash) and hypotension (1.5\%) as adverse events post IVP administration.

This study included a relatively large sample size of both allogeneic and autologous adult HSCT patients, which is larger than previously reported studies. However, our study had several limitations. One is being a retrospective study and therefore there is a potential of missing some of data especially concerning adverse drug reactions. Secondly, the adverse drug reactions were identified through the pharmacy documentation reporting system and electronic patient profiles, and thus some might have been missed due to underreporting. Another limitation to our study is related to the diagnostics used, since quantitative real time PCR was not done in our patients due to its unavailability at our center. This may be a limitation as PCR is considered to have better sensitivity in immune compromised patients, compared to the microscopic examination used in our patients [21]..

\section{Conclusions}

Our findings support the use of IVP for PCP prophylaxis in adult HSCT patients. Prospective studies should be conducted to confirm the efficacy and tolerability of IVP.

\section{Abbreviations \\ TMP/SMX: Trimethoprim/sulfamethoxazole; IVP: Intravenous pentamidine; KHCC: King Hussein Cancer Center; IV: Intravenous; HSCT: Hematopoietic stem cell transplantation; GVHD: Graft versus host disease}

\section{Acknowledgements}

The authors thank Dr. Lama Nazer, PharmD, BCPS, King Hussein Cancer Center, who reviewed the manuscript, and Nilly Hussein, Data Manger of the Blood and Marrow Transplantation Program at King Hussein Cancer Center, for her assistance in obtaining the data from the bone marrow transplant electronic database.

\section{Authors' contributions}

WA and RN developed the research question and the research protocol. WA, AA, NA and RN were involved in data collection, data analysis, interpretation of the results and writing the manuscript. All authors reviewed and approved the final manuscript.

\section{Funding}

Not applicable.

\section{Availability of data and materials}

The datasets used and/or analysed during the current study are available from the corresponding author on reasonable request.

\section{Ethics approval and consent to participate}

The study protocol was approved by the Institutional Review Board of King Hussein Cancer Center, Amman-Jordan under the reference number of 19KHCC51, and the Institutional Review Board granted the permission to access the raw data and patients' electronic medical records. The raw data that was obtained from the bone marrow transplant unit database and pharmacy billing system was identified to the corresponding authors: WA, RN, NA and AA for data collection. The included data was then coded and was anonymous to the data analysis personnel. A waiver of consent was submitted to access the retrospective data.

Consent for publication

Not applicable. 


\section{Competing interests}

The authors declare that they have no competing interests.

Received: 19 February 2020 Accepted: 28 May 2020

Published online: 05 June 2020

\section{References}

1. Bennett JE, Dolin R, Blaser MJ. Mandell, Douglas, and Bennett's Principles and Practice of Infectious Diseases, 7th edition. Elsevier Inc:; 2010.

2. Thomas CF Jr, Limper AH. Pneumocystis pneumonia. N Engl J Med. 2004; 350:2487-98.

3. Diri R, Anwer F, Yeager A, Krishnadasan R, McBride A. Retrospective review of intravenous pentamidine for Pneumocystis pneumonia prophylaxis in allogeneic hematopoietic stem cell transplantation. Transpl Infect Dis. 2016; 18:63-9.

4. De Castro N, Neuville S, Sarfati C, Ribaud P, Derouin F, Gluckman E, et al. Occurrence of Pneumocystis jiroveci pneumonia after allogeneic stem cell transplantation: a 6-year retrospective study. Bone Marrow Transplant. 2005;36(10):879-83.

5. Tuan IZ, Dennison D, Weisdorf DJ. Pneumocystis carinii pneumonitis following bone marrow transplantation. Bone Marrow Transplant. 1992; 10(3):267-72.

6. Centers for Disease Control and Prevention; Infectious Disease Society of America; American Society of Blood and Marrow Transplantation. Guidelines for preventing opportunistic infections among hematopoietic stem cell transplant recipients. MMWR Recomm Rep. 2000;49(RR-10):1-125.

7. Maertens J, Cesaro S, Maschmeyer G, Einsele H, Donnelly JP, Alanio A, et al. ECIL guidelines for preventing Pneumocystis jirovecii pneumonia in patients with haematological malignancies and stem cell transplant recipients. J Antimicrob Chemother. 2016;71(9):1-8.

8. Tomblyn M, Chiller T, Einsele H, Gress R, Sepkowitz K, Storek J, et al. Guidelines for preventing infectious complications among hematopoietic cell transplant recipients: a global perspective. Biol Blood Marrow Transplant. 2009;15(10):1143-238.

9. National Comprehensive Cancer Network. Prevention and Treatment of Cancer-related Infections [Version.1.2020]. (Available from: https://www.nccn. org/professionals/physician gls/pdf/infections.pdf, Accessed 24 Jan 2020).

10. Vasconcelles MJ, Bernardo MV, King C, Weller EA, Antin JH. Aerosolized pentamidine as pneumocystis prophylaxis after bone marrow transplantation is inferior to other regimens and is associated with decreased survival and an increased risk of other infections. Biol Blood Marrow Transplant. 2000;6(1):35-43.

11. Kim SY, Dabb AA, Glenn JD, Snyder KM, Chuk MK, Loeb DM. Intravenous pentamidine is effective as second line pneumocystis pneumonia prophylaxis in pediatric oncology patients. Pediatric Blood Cancer. 2008; 50(4):779-83.

12. Williams KM, Ahn KW, Chen M, Aljurf MD, Agwu AL, Chen AR, et al. The incidence, mortality and timing of Pneumocystis jiroveci pneumonia after hematopoietic cell transplantation: a CIBMTR analysis. Bone Marrow Transplant. 2016;51(4):573-80.

13. Curi DA, Duerst RE, Badke C, Bell J, Chaudhury S, Kletzel M, et al. IV pentamidine for Pneumocystis jiroveci pneumonia prophylaxis in pediatric allogeneic stem cell transplant patients. Bone Marrow Transplant. 2016; 51(10):1394-6

14. Solodokin $L$, Klejmont LM, Scipione MR, Dubrovskaya Y, Lighter-Fisher J, Papadopoulos J. Safety and effectiveness of intravenous pentamidine for prophylaxis of Pneumocystis jirovecii pneumonia in pediatric hematology/ oncology patients. J Pediatr Hematol Oncol. 2016;38(6):e180-5.

15. Burroughs G, Connor T, McDiarmid M. NIOSH alert: preventing occupational exposures to antineoplastic and other hazardous drugs in health care settings DHHS (NIOSH) (Publ. No. 2004-165). Cincinnati; 2004. p. 58. Available from: https://www.cdc.gov/niosh/docs/2004-165/pdfs/2004-165. pdf, Accessed 24 Jan 2020].

16. Sweiss K, Anderson J, Wirth S, Oh A, Quigley JG, Khan I, et al. A prospective study of intravenous pentamidine for PJP prophylaxis in adult patients undergoing intensive chemotherapy or hematopoietic stem cell transplant. Bone Marrow Transplant. 2018;53(3):300-6.

17. Quinn M, Fannin JT, Sciasci J, Bragg A, Campbell PK, Carias D, et al. Pentamidine for prophylaxis against Pneumocystis jirovecii pneumonia in pediatric oncology patients receiving immunosuppressive chemotherapy. Antimicrob Agents Chemother. 2018;62(8):e00173.
18. DeMasi J, Cox J, Leonard D, Koh A, Aquino V. Intravenous pentamidine is safe and effective as primary Pneumocystis pneumonia prophylaxis in children and adolescents undergoing hematopoietic stem cell transplantation. Pediatr Infect Dis J. 2013;32(9):933-6.

19. Drug information: pentamidine (systemic). Lexicomp: http://online.lexi.com/ Ico/action/doc/retrieve/docid/multinat_f/5873117?cesid= 7FVU8wrSNpB\&searchUrl=\%2Flco\%2Faction\%2Fsearch\%3Fq\%3 DPentamidine\%2520\%26t\%3Dname\%26va\%3DPentamidine\%2520\#adr. Accessed 5 Apr 2020.

20. Drug information: Intravenous pentamidine. Electronic medicines compendium: https://www.medicines.org.uk/emc/product/977. Accessed 5 Apr 2020

21. Robert-Gangneux F, Belaz S, Revest M, Tattevin P, Jouneau S, Decaux O, et al. Diagnosis of Pneumocystis jirovecii pneumonia in immunocompromised patients by real-time PCR: a 4-year prospective study. J Clin Microbiol. 2014:52(9):3370-6.

\section{Publisher's Note}

Springer Nature remains neutral with regard to jurisdictional claims in published maps and institutional affiliations.

\section{Ready to submit your research? Choose BMC and benefit from:}

- fast, convenient online submission

- thorough peer review by experienced researchers in your field

- rapid publication on acceptance

- support for research data, including large and complex data types

- gold Open Access which fosters wider collaboration and increased citations

- maximum visibility for your research: over $100 \mathrm{M}$ website views per year

At BMC, research is always in progress.

Learn more biomedcentral.com/submissions 